\section{AB0391 LOW SERUM COMPLEMENT C3 LEVEL AS A RISK FACTOR FOR RELAPSE OF ANTINEUTROPHIL CYTOPLASMIC ANTIBODY-ASSOCIATED VASCULITIS: A RETROSPECTIVE COHORT STUDY}

H. Sakai ${ }^{1}$, H. Yamashita ${ }^{1}$, S. Nakajima ${ }^{1}$, Y. Takahashi ${ }^{1}$, H. Kaneko ${ }^{1} .{ }^{1}$ National Center for Global Health and Medicine, Division of Rheumatic Diseases, Tokyo, Japan

Background: The alternative pathway of complement activation has recently been recognized as a key pathogenic event in antineutrophil cytoplasmic antibody (ANCA)-associated vasculitis (AAV). Some previous studies have reported that low serum complement C3 level in AAV patients is associated with more severe renal disease, worse renal prognosis, or higher mortality. However, the correlation between low serum $\mathrm{C} 3$ level and AAV relapse remains unclear.

Objectives: To analyze the clinical characteristics and outcomes of AAV patients with low serum $\mathrm{C} 3$ levels at the time of diagnosis.

Methods: We conducted a retrospective observational cohort study including 83 consecutive patients diagnosed with AAV in our hospital from January 1999 to December 2020. Serum C3 levels were measured at diagnosis. AAV included microscopic polyangiitis (MPA), granulomatosis with polyangiitis (GPA), and eosinophilic granulomatosis with polyangiitis (EGPA); patients with ANCA-negative AAV were excluded. Patients were divided into low- and high-C3 groups (C3 $<100$ and $\geq 100 \mathrm{mg} / \mathrm{dL}$, respectively). We compared the clinical characteristics, and relapse-free and overall survival rates, of the two groups, and identified predictors of AAV relapse.

Results: Of the 83 patients (MPA, $n=61$; GPA, $n=18$; EGPA, $n=4), 20(24 \%)$ were in the low-C3 group. We found no significant group difference in sex, body mass index, disease type, ANCA subtype, Birmingham Vasculitis Activity Score (BVAS), or treatment. The low-C3 group patients were older $(p=0.01)$, and had a higher Five Factor Score (FFS) $(p=0.01)$ and a lower remission rate $(p=0.02)$, than the high-C3 group. The generalized Wilcoxon test revealed that the relapsefree survival time was significantly shorter in the low-C3 group (29 months; $95 \%$ confidence interval [Cl]: 15-49) than in the high-C3 group (82 months; $95 \% \mathrm{Cl}$ $61-N A ; p=0.01$ ) (Figure 1A). The overall survival was also shorter in the low-C3 group (83 months; $95 \% \mathrm{Cl}$ : 8-121) than in the high-C3 group (112 months; $95 \%$ Cl: 77-NA; $p=0.03$ ) (Figure 1B). In the Cox proportional hazards model, a low C3 level (<100 mg/dL) (hazard ratio [HR], 3.01; 95\% Cl: 1.29-7.04], $\mathrm{p}=0.01$ ) and GPA (HR, 3.04; 95\% Cl: 1.32-7.01; $p=0.01$ ) were independent predictors of AAV relapse.
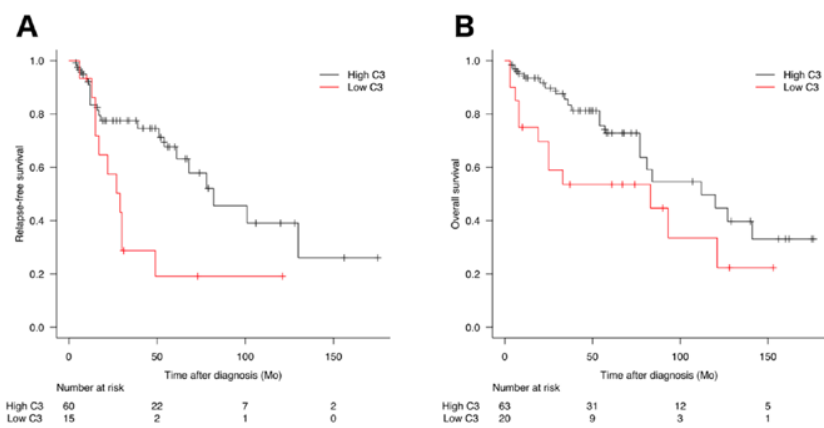

Figure 1. Kaplan-Meier estimates of the relapse-free (A) and overall (B) survival rates of AAV patients by baseline serum C3 levels. Eight patients who did not show remission were excluded in the relapse-free survival analysis. Black line: high-C3 group ( $\geq 100 \mathrm{mg} / \mathrm{dL}$ ); red line: low-C3 group $(<100 \mathrm{mg} / \mathrm{dL})$.

Conclusion: AAV patients with low C3 levels at diagnosis were at higher risk of relapse. Larger prospective studies are required to confirm these findings.

Disclosure of Interests: None declared

DOI: 10.1136/annrheumdis-2021-eular.1517

\section{AB0392 1 PRELIMINARY VALIDATION OF THE ITALIAN VERSION OF ANCA-ASSOCIATED VASCULITIS PATIENT-REPORTED OUTCOME (AAV-PRO_ITA) QUESTIONNAIRE: FATIGUE AND CHRONIC PAIN AS UNMET NEEDS BY CURRENT TREATMENTS}

E. Treppo ${ }^{1}$, A. Palese ${ }^{2}$, S. De Vita ${ }^{1}$, L. Quartuccio ${ }^{1} .{ }^{1}$ University of Udine, Rheumatology Clinic, Udine, Italy; ${ }^{2}$ School of Nursing, University of Udine, Department of Medicine, Udine, Italy

Background: The ANCA-associated vasculitis patient-reported outcome (AAVPRO) questionnaire is a 29-item disease-specific PRO measure for AAV (1), proving to be valid, reliable, feasible, and able to discriminate among disease states.
Objectives: The purpose of this study is to validate the Italian version of AAVPRO questionnaire (AAV-PRO_ita).

Methods: AAV-PRO has been translated in collaboration with Oxford and Bristo University (UK). Then, consecutive Italian-speaking AAV-patients were collected in order to validate the questionnaire. Inclusion criteria are AAV diagnosis (ANCA positivity at least one time and/or biopsy-proven AAV), and age $\geq 18$ years old Participants completed the AAV-PRO_ita three different times: at baseline, after 5-7 days, and at month 3. Friedman test was applied to verify internal consistency of the questionnaire over time.

Results: 27 AAV-patients (16 females, 11 males) were recruited and completed the questionnaire at baseline, after 5-7 days, and at month 3 . The main diagnosis was granulomatosis with polyangioitis (GPA) (19/27, 70.4\%), following by eosinophilic granulomatosis with polyangioitis (EGPA) (8/27, 29.6\%). Mean (SD) BVASv3 was 1 (2) at baseline, and it was unchanged at month 3 in 25/27 $(92.6 \%)$. The mean age at onset of disease was $54.7 \pm 17.3$ years and the median disease duration was 51 months (1-3 interquartile range 34-76). Their history was characterized by at least one relapse in 10/27 (37\%) patients, at least one hospitalization due to AAV in 19/27 (70.4\%), and ESRD in 1/27 (3.7\%). At the last follow up, $24 / 27$ (88.9\%) of patients were taking an immunosuppressive drug [rituximab (8/24), azathioprine (8/24), methotrexate (7/24) or mycophenolate mofetil (1/24)] and more than two-thirds were also taking glucocorticoids (19/27, $70.3 \%$ ). Fatigue (item 9) and arthralgia (item 6) were the most important symptoms, and half of the patients $(14 / 27,51.9 \%)$ classified them as "moderate" or "severe". Nearly one-third of patients (8/27, 29.6\%) admitted to being "usually" or "always" concerned about their future (item 19) and long-term therapy (item 29) (Figure 1). Friedman test was not significant ( $p>0.05$ ), indicating internal consistency of the questionnaire. A large multicentre Italian study has been organized based on this preliminary data coming from the coordinating centre, and it is now recruiting. The results will be soon available by the Italian Vasculitis Study Group. Conclusion: The AAV-PRO ita is a new disease-specific PRO measure for AAV. It is a self-administered questionnaire and could become an important tool to evaluate health-related quality of life in AAV (2). As in other systemic autoimmune diseases, fatigue and chronic pain represent an unmet need to be addressed by future treatment strategies. REFERENCES:

[1] J. C. Robson et al., «Validation of the ANCA-associated vasculitis patient-reported outcomes (AAV-PRO) questionnaire», Ann. Rheum. Dis., 2018, vol. 77, n. 8, p. 1157-1164, doi: 10.1136/annrheumdis-2017-212713.

[2] L. Quartuccio, et al., «Healthcare and economic burden of ANCA-associated vasculitis in Italy: an integrated analysis from clinical and administrative databases», Intern Emerg Med, July 2020, doi: 10.1007/s11739-020-02431-y.

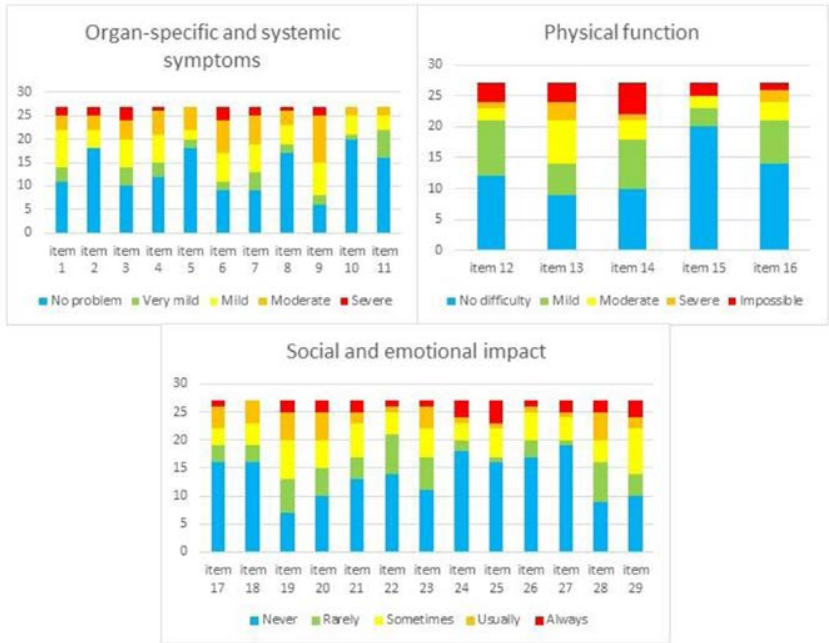

Figure 1.

Disclosure of Interests: None declared DOI: 10.1136/annrheumdis-2021-eular.2123

\section{AB0393 PRE-EXISTING MORBIDITY AS A RISK FACTORS FOR MORTALITY IN IGA VASCULITIS}

J. Nossent $^{1,2}$, D. Preen ${ }^{3}$, W. Raymond ${ }^{1}$, H. Keen ${ }^{3,4}$, C. Inderjeeth ${ }^{1,2}{ }^{1}$ The University of Western Australia, Medical School, Rheumatology Section, Perth, Australia; ${ }^{2}$ Sir Charles Gairdner Hospital, Rheumatology, Nedlands, Australia; ${ }^{3}$ The University of Western Australia, School for Population and Global health, Perth, Australia; ${ }^{4}$ Fiona Stanley Hospital, Rheumatology, Murdoch, Australia

Background: IgA vasculitis is generally considered to be a self-limiting condition, but this is at odds with the increased mortality observed in adult patients with IgA vasculitis (1) 
Objectives: With sparse data on prognostic factors in IgAV, we investigated whether pre-existing conditions are risk factors for mortality in adult IgAV patients. Methods: Observational population-based cohort study using state-wide linked longitudinal health data for adults with IgAV $(n=267)$ and matched controls $(n=1080)$ between 1980-2015. Charlson comorbidity index $(\mathrm{CCl})$ and serious infections (SI) were recorded over an extensive lookback period prior to diagnosis. Date and causes of death were extracted from the WA Death Registry. Mortality rate (deaths/1000 person-years) ratios (MRR) and time dependent survival analysis assessed the risk of death. Age and gender specific mortality rate data were obtained from the Australian Bureau of Statistics.

Results: During $9.9( \pm 9.8)$ years lookback IgAV patients accrued higher $\mathrm{CCl}$ scores $(2.60$ vs1.50 p<0.001) and had higher risk of SI (OR 8.4, p<0.001), not fully explained by $\mathrm{CCl}$ scores. During 19 years follow-up, the risk of death in IgAV patients $(n=137)$ was higher than in controls $(n=397)$ (MRR 2.06, $\mathrm{Cl} 1.70$ $2.50, \mathrm{p}<0.01$ ) and the general population (SMRR 5.64, Cl 4.25, 7.53, p<0.001). Survival in IgAV was reduced at five (72.7 vs. $89.7 \%)$ and twenty years $(45.2 \%$ vs. $65.6 \%$ ) (both $\mathrm{p}<0.05)$. $\mathrm{CCl}$ (HR1.88, $\mathrm{Cl}: 1.25$ - 2.73, $\mathrm{p}=0.001)$, renal failure (HR 1.48, Cl: $1.04-2.22, p=0.03$ ) and prior SI (HR 1.48, Cl:1.01 - 2.16, p=0.04) were independent risk factors. Death from infections (5.8 vs $1.8 \%, p=0.02)$ was significantly more frequent in IgAV patients.

Conclusion: Premorbid accrual of comorbidity is increased and predicts premature death in IgAV patients. However, comorbidity does not fully explain the increased risk of serious infections prior to diagnosis or the increased mortality due to infections in IgAV.

REFERENCES:

[1] Villatoro-Villar M, Crowson CS, Warrington KJ, Makol A, Ytterberg SR, Koster

MJ. Clinical Characteristics of Biopsy-Proven IgA Vasculitis in Children and

Adults: A Retrospective Cohort Study. Mayo Clin Proc. 2019;94(9):1769-80. Acknowledgements: The authors would like to acknowledge the support of the Arthritis Foundation of WA and acknowledge the Western Australian Data Linkage Branch, the Western Australian Department of Health, and the data custodians of, the Hospital and Morbidity Data Collection, the Emergency Department Data Collection the WA Cancer Register and the WA Death Register for their assistance with the study.

Disclosure of Interests: None declared

DOI: 10.1136/annrheumdis-2021-eular.2307

\section{AB0394 CLINICAL CHARACTERISTICS OF EOSINOPHILIC GRANULOMATOSIS WITH POLYANGIITIS: A SINGLE- CENTER RETROSPECTIVE ANALYSIS ON 52 CASES OF CHINESE PATIENTS}

L. F. Chen ${ }^{1}$, Y. Mo ${ }^{1}$, Q. H. Li ${ }^{1}$, D. H. Zheng ${ }^{1}$, L. Dai ${ }^{1}{ }^{1}$ Sun Yat-Sen Memorial Hospital, Sun Yat-Sen University, Rheumatology, Guangzhou, China

Background: Eosinophilic granulomatosis with polyangiitis (EGPA) is a rare and heterogeneous systemic vasculitis. Different patients or the same patient in different stages show different manifestations, which may lead to misdiagnosis and delay treatment.

Objectives: To analyze the clinical characteristics in Chinese patients with EGPA.

Methods: EGPA patients who fulfilled the 1990 ACR classification criteria were included between December 2003 and April 2020. The demographic and clinical characteristics were collected and analyzed retrospectively.

Results: There were 52 EGPA patients recruited, $34(65.4 \%)$ patients were males and the median age at onset was $47(38 \sim 55)$ years. The median duration from disease onset to diagnosis was 30(4 96) months. For initial symptoms, respiratory manifestations $(61.5 \%)$ were the most common, including $42.3 \%$ patients beginning with asthma, followed by $21.2 \%$ with nose/paranasal sinuses manifestations. Respiratory medicine $(53.8 \%)$ were the most common department at first visit, followed by rheumatology medicine (11.5\%, Figure $1 \mathrm{~A})$. There were $44.2 \%$ EGPA patients definitely diagnosed at the department of rheumatology or after consultation by rheumatologists.

During the whole disease process, the most common clinical manifestations were asthma (88.5\%), then nose/ paranasal sinuses (84.6\%), pulmonary $(76.9 \%)$ and nervous system $(61.5 \%)$ manifestations, followed by constitutional symptom $(44.2 \%)$, heart $(36.5 \%)$ and skin $(23.1 \%)$ involvement. Only $9.6 \%$ patients had gastrointestinal tract involvement and $3.8 \%$ had renal involvement (Figure 1B). There were $46(88.5 \%)$ patients showing the ratio of peripheral blood eosinophils $>10 \%$ at diagnosis. Among the rest 6 patients, 3 had higher eosinophil ratio before diagnosis, while the other 3 patients had been treated with glucocorticoid before diagnosis, of whom 2 patients showed pathological eosinophil infiltration in lung or paranasal sinuses mucosa, respectively. There were $8(15.4 \%)$ patients with positive ANCA. Compared with EGPA patients with negative ANCA, they had lower incidence of asthma (62.5\% vs. 93.2\%), but higher incidence of constitutional symptoms $(87.5 \%$ vs. $36.4 \%)$, arthralgia $(50.0 \%$ vs. $6.8 \%)$ and renal involvement $(25.0 \%$ vs. $0.0 \%)$, higher peripheral eosinophil count $\left[2.06(0.80 \sim 4.51) \times 10^{9} / \mathrm{L}\right.$ vs. $\left.1.81(0.93 \sim 3.32) \times 10^{9} / \mathrm{L}\right]$, ESR $[20(7 \sim 77) \mathrm{mm} / \mathrm{h}$ vs.
18(9 42) $\mathrm{mm} / \mathrm{h}$ ] and CRP [18.5(3.2 65.9) mg/L vs. 3.3(3.2 13.0) mg/L], higher Birmingham vasculitis activity score $[17(10 \sim 22)$ vs. 13(9 15)] and vasculitis damage index $[3(1 \sim 3)$ vs. 2(1 2), all $P<0.05]$. There were $21.2 \%$ EGPA patients showing poor prognostic factors according to five-factor score (FFS) and $34.6 \%$ patients according to the revised FFS.

Conclusion: EGPA patients may have no asthma especially those with positive ANCA. Multi-disciplinary collaboration especially based on rheumatologists and pulmonologists should be emphasized for early identification and prompt treatment.
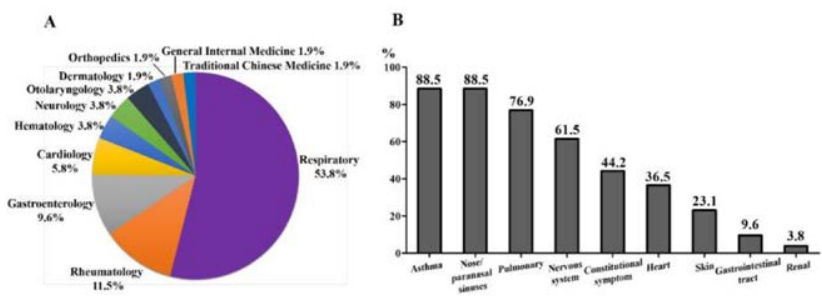

Figure 1. Department at first visit (A) and clinical manifestations during the whole disease process of 52 Chinese EGPA patients(B)

Disclosure of Interests: None declared

DOI: 10.1136/annrheumdis-2021-eular.2359

\section{AB0395 RENAL INVOLVEMENT IN HENOCH-SCHÖNLEIN PURPURA}

N. Ben Chekaya ${ }^{1}$, S. Daada ${ }^{1}$, M. Brahim ${ }^{1}$, I. Chaabene ${ }^{1}$, R. Klii ${ }^{1}$, M. Kechida ${ }^{1}$ S. Hammami ${ }^{1}{ }^{1}$ Fattouma Bourguiba Hospital, Internal Medicine and Endocrinology Departement, Monastir, Tunisia

Background: Henoch-Schönlein purpura (HSP), also known as rheumatic purpura, is an immune complex vasculitis affecting small vessels with dominant IgA deposits. Clinical manifestations mainly involve cutaneous purpura, arthralgias and/or arthritis, acute enteritis and glomerulonephritis. HSP is more common among children than adults. Kidney damage is the principal prognostic determinant in HSP. The aim of this study is to evaluate the renal manifestations of HSP.

Objectives: The aim of this study is to evaluate the renal manifestations of HSP.

Methods: This was a retrospective study of 35 patients with diagnosed HSA who were admitted between the time period may 2008 and november 2018. All of these patients met the American College of Rheumatology classification criteria for a diagnosis of HSP. Renal involvement was defined as the presence of proteinuria or hematuria.

Results: The study group consisted of 35 patients, 20 women (57\%) and 15 men (43\%). The mean age was $48.8 \pm 18.7$ years. The incidence rates of skin, joint, gastrointestinal and central nervous system involvement were 91.4, 77.1 54.2 and $2.8 \%$, respectively. Elevated serum IgA levels occurred in $5.7 \%$. Six patients $(17.1 \%)$ were given corticosteroids during the first 3 months of their HSP, which were indicated for severe gastrointestinal $(n=5)$ or renal involvement $(n=1)$. Eighteen of the thirty five patients $(51.42 \%)$ had renal involve ment. There were 12 women and six men. All patients had proteinuria (100\%). Hematuria has been identified in 11 patients $(61.1 \%)$ and renal insufficiency in 4 patients $(22.2 \%)$. The renal involvement was mainly detected in the first month. Renal biopsy was affected in four patients (22.2\%). An endocapillary glomerulonephritis was found in 3 patients and an extra capillary necrotizing glomerulonephritis in one patient. The immunofluorescence showed the presence of IgA deposits in 4 patients.

Conclusion: Renal involvement dominates the prognosis for the disease. The most common clinical expression is the association of microscopic hematuria with proteinuria.

Disclosure of Interests: None declared

DOI: 10.1136/annrheumdis-2021-eular.2670

\section{AB0396 RAPID IMPROVEMENT IN CYSTOID MACULAR EDEMA WITH HIGH DOSE INTRAVENOUS METHYLPREDNISOLONE IN NON-INFECTIOUS UVEITIS OF DIFFERENT IMMUNE MEDIATED INFLAMMATORY DISEASES}

N. Vegas-Revenga ${ }^{1}$, J. L. Martín-Varillas ${ }^{2}$, V. Calvo-Río ${ }^{3}$, I. González-Mazón ${ }^{3}$ L. Sanchez-Bilbao ${ }^{3}$, E. Beltrán ${ }^{4}$, A. Fonollosa ${ }^{5}$, O. Maiz-Alonso ${ }^{6}$, A. Blanco ${ }^{7}$, M. Cordero-Coma ${ }^{8}$, N. Ortego ${ }^{9}$, I. Torre-Salaberri ${ }^{10}$, F. Francisco ${ }^{11}$, S. Muñoz Fernandez $^{12}$, M. D. M. Esteban-Ortega ${ }^{13}$, M. Díaz-Llopis ${ }^{14}$, J. Cañal $^{15}, \mathrm{~J}$ A. Ventosa ${ }^{15}$, R. Demetrio-Pablo ${ }^{15}$, L. Domínguez ${ }^{16}$, M. Agudo-Bilbao ${ }^{3}$, 